Grav. Cosmol. No. 2, 2011

\title{
Gravitation as an effect of nonlinear electrodynamics ${ }^{1}$
}

\author{
Alexander A. Chernitskii ${ }^{2}$ \\ Friedmann Laboratory of Theoretical Physics, St. Petersburg, Russia; \\ State University of Engineering and Economics, ul. Marata 27, St. Petersburg 191002, Russia
}

Received October 4, 2010

\begin{abstract}
We consider an approach to unification of the gravitational and electromagnetic interactions based on the existence of an effective Riemannian space in nonlinear electrodynamics. In the context of this approach, we discuss the possibility of screening the gravitational field.
\end{abstract}

\section{Introduction}

The author's works $[1,2]$ have suggested an approach to solving the problem of unification of gravitation and electromagnetism on the basis of the existence of an effective Riemannian space for interacting solitons in nonlinear electrodynamics. In [1], Mie's model of nonlinear electrodynamics was considered, and in [2] that of Born-Infeld. A characteristic feature of the present approach to the unification problem is that gravitation is secondary with respect to electromagnetism, or one can say that it is induced by nonlinear electromagnetism.

Different aspects of this approach have been discussed in [3-7] and elsewhere.

Under such an approach, it is natural to expect that some aspects of electromagnetism should manifest themselves in gravitation as well. In particular, one can raise the question of screening the gravitational field similar to the well-known electromagnetic field screening.

\section{The field model and electro- magnetic particles}

As the field of the model, we take the 4-vector field $A_{\mu}$ (Greek indices take the values $0,1,2,3$ ), since it is this vector with respect to which the action functional is varied in electrodynamics. The elec-

\footnotetext{
${ }^{1}$ Talk given at the International Conference RUDN-10, June 28 - July 3, 2010, PFUR, Moscow

${ }^{2}$ e-mail: AAChernitskii@mail.ru, AAChernitskii@engec.ru
}

tromagnetic tensor is defined in the usual way,

$$
F_{\mu \nu}=\frac{\partial A_{\nu}}{\partial x^{\mu}}-\frac{\partial A_{\mu}}{\partial x^{\nu}}
$$

As the variational principle of the model, the following generally covariant variational principle is taken:

$$
\begin{aligned}
& \delta \int \sqrt{\left|\operatorname{det}\left(G_{\mu \nu}\right)\right|}(\mathrm{d} x)^{4}=0, \\
& G_{\mu \nu}=g_{\mu \nu}+\chi^{2} F_{\mu \nu} .
\end{aligned}
$$

where $g_{\mu \nu}$ is the metric tensor of flat space-time, $(\mathrm{d} x)^{4} \equiv \mathrm{d} x^{0} \mathrm{~d} x^{1} \mathrm{~d} x^{2} \mathrm{~d} x^{3}$, and $\chi$ is a dimensionful constant.

This variational principle was considered in a somewhat more general form by A.S. Eddington [8] and A. Einstein [9]. M. Born and L. Infeld [10] have considered an electrodynamic model corresponding to the variational principle (2).

The set of equations obtained from the variational principle (2) has the form

$$
\frac{\partial}{\partial x^{\mu}} \sqrt{|g|} f^{\mu \nu}=0
$$

where

$$
\begin{aligned}
& f^{\mu \nu} \equiv \frac{\chi^{-2} \partial \mathcal{L}}{\partial\left(\partial_{\mu} A_{\nu}\right)}=\frac{1}{\mathcal{L}}\left(F^{\mu \nu}-\frac{\chi^{2}}{2} \mathcal{J} \varepsilon^{\mu \nu \sigma \rho} \mathcal{F}_{\sigma \rho}\right), \\
& \mathcal{L} \equiv \sqrt{\left|1-\chi^{2} \mathcal{I}-\chi^{4} \mathcal{J}^{2}\right|} \\
& \mathcal{I} \equiv F_{\mu \nu} F^{\nu \mu} / 2, \\
& \mathcal{J} \equiv \varepsilon_{\mu \nu \sigma \rho} F^{\mu \nu} F^{\sigma \rho} / 8, \\
& \varepsilon_{0123} \equiv \sqrt{|g|}, \quad \varepsilon^{0123}=-1 / \sqrt{|g|} .
\end{aligned}
$$


The symmetric stress-energy tensor of the model has the form

$$
T_{. \nu}^{\mu} \equiv\left(f^{\mu \rho} F_{\nu \rho}-\chi^{-2}(\mathcal{L}-1) \delta_{\nu}^{\mu}\right) / 4 \pi .
$$

The characteristics equation in this model has the following remarkable form:

$$
\tilde{g}^{\mu \nu} \frac{\partial \Phi}{\partial x^{\mu}} \frac{\partial \Phi}{\partial x^{\nu}}=0
$$

where $\Phi\left(x^{\mu}\right)=0$ is the equation of the characteristic surface,

$$
\tilde{g}^{\mu \nu} \equiv g^{\mu \nu}-4 \pi \chi^{2} T^{\mu \nu} .
$$

Here $T^{\mu \nu}$ is the stress-energy tensor of the form (5).

The set of equations in this model has spatially localized solutions which are attributed to electromagnetic particles. The simplest solution of this kind, possessing spherical symmetry, has been considered by Born and Infeld as a model of the electron [9]. This solution has a finite total energy (mass). However, since the spherically symmetric solution does not possess a magnetic moment and a total angular momentum (spin), it cannot be related to the real electron. More complex solutions with axial symmetry can have a mass, a spin and a magnetic moment $[2,12]$.

In addition to a static part, particlelike solutions should have a rapidly oscillating part responsible for the wave properties of particle and, as it turns out in the present approach, for the gravitational interaction [13].

\section{The effective Riemannian space and gravitation}

In an investigation of a multiparticle solution using a perturbation method, one takes as an initial approximation a sum of one-particle solutions with trajectories determined from the condition of total momentum conservation in the localization domains of solitonic particles [2]. $\mathrm{n}$ this way one obtains the electromagnetic interaction of solitonic particles. This interaction originates from the static part of the solitonic solution (in its own reference frame).

To explain the gravitational interaction in this approach, it is necessary to take into account the rapidly oscillating part of the soliton under study and the field of remote solitons. In addition, it is necessary to take into account the rapidly oscillating electromagnetic background.

In the initial approximation, the light wave and the rapidly oscillating part of a solitonic particle propagate along geodesics of the effective Riemannian space with the metric $\tilde{g}^{\mu \nu}$, depending on the field of remote solitons. This follows from the characteristic equation (6) [2].

In the initial approximation, the static part and the rapidly oscillating part of a massive solitonic particle are connected. In this way, the static part is as though dragged by the rapidly oscillating part.

For a correct behavior of the effective metric (i.e., in order to obtain the Newtonian potential), the radiation background must be taken into consideration $[3,13]$.

Thus the gravitational interaction is stipulated by the rapidly oscillating component of solitonic solutions.

\section{On possible screening of gravity}

Since in the framework of the present approach the gravitational interaction is a manifestation of the electromagnetic field nonlinearity, it is logical to consider the opportunity of screening the gravitational field similar to electromagnetism. Herewith, since gravity is related to the rapidly oscillating part of the solitonic solutions, one should consider a shield for electromagnetic waves as a prototype gravitational screen.

As shown in [13], the Newtonian potential in the effective metric created by a certain soliton can emerge due to an interaction between the rapidly oscillating part of the soliton with the radiation background. If one places the soliton into a perfectly reflecting sphere, there will be no interaction between the soliton and the radiation background. It should be noted that outside the sphere the radiation background will excite the corresponding spherical modes of standing electromagnetic waves. Their interaction with the background can again give a Newtonian potential in the effective metric. However, if we suppose that the radiation background has a sufficiently small magnitude, then the gravitational field outside the sphere will be weaker than if the spherical screen were absent.

Moreover, if one places a plane plate of finite 
size closely to a soliton, then, in the shade region for the solitonic radiation (i.e., for the rapidly oscillating part of the solution), one can also expect weakening of the soliton's gravitational field.

Of interest is certainly the question of the existence of substances from which one could fabricate such a reflecting screen. To answer it, one should above all determine the order of magnitude of the frequency of the rapidly oscillating parts of solitonic solutions corresponding to real particles. This frequency is determined from the well-known relations of quantum physics:

$$
m c^{2}=h \nu \quad \Longrightarrow \quad \nu=\frac{m c^{2}}{h}=\frac{c}{\lambda_{C}},
$$

where $m$ is the particle mass, $c$ is the speed of light in vacuum, $h$ is the Planck constant, $\nu$ is the frequency, and $\lambda_{C}$ is the Compton wavelength of the particle.

Bearing in mind the Compton wavelengths of the electron $\left(\approx 2.4 \cdot 10^{-3} \mathrm{~nm}\right)$ and proton $(\approx 1.3$. $\left.10^{-6} \mathrm{~nm}\right)$, let us estimate the frequency:

$$
\nu \sim 10^{20} \div 10^{23}(\mathrm{~Hz}) .
$$

Such frequencies and the corresponding electromagnetic wavelengths are characteristic of gamma radiation $\left(\lambda<5 \cdot 10^{-3} \mathrm{~nm}\right)$.

Thus to obtain a gravitational screen in the present approach it is necessary to have a mirror for gamma rays.

Gamma rays are known to possess a high penetrating power in usual substances, and creating such a mirror can now seem to be an unreal idea. One should not, however, exclude the opportunity of obtaining, say, a nano-material that would possess the necessary properties.

\section{Conclusion}

In the framework of the theory under consideration, there is in principle an opportunity of a gravitational screening effect. Such screening may be realized with the aid of materials with unusual properties.

From this viewpoint, it seems to be expedient to carry out the corresponding experimental tests of newly obtained (nano-)materials for the necessary properties (from the viewpoint of the present approach) for observing the gravitational screening effect.

\section{References}

[1] A. A. Chernitskii. Long-range interaction of fourvector field solitons of the Minkowskian space, Theoret. Math. Phys. 90 (3), 260 (380 in Russian) (1992).

[2] A. A. Chernitskii, Dyons and interactions in nonlinear (Born-Infeld) electrodynamics, J. High Energy Phys. 12, Paper 10 (1999); hep-th/9911093.

[3] A. A. Chernitskii, Induced gravitation as nonlinear electrodynamics effect, Grav. Cosmol. 8, Supplement, 157-160 (2002); gr-qc/0211034.

[4] A. A. Chernitskii, Born-Infeld equations. In: Encyclopedia of Nonlinear Science, ed. A. Scott, (Routledge, New York — London, 2004), 67-69; hepth/0509087.

[5] A. A. Chernitskii, Gravitation and electromagnetism in a theory of a unified four-vector field, Grav. Cosmol. 12, 130-132 (2006); hepth/0609204.

[6] A. A. Chernitskii, Gravitation as a vacuum nonlinear electrodynamics effect. In: On Recent Developments in Theoretical and Experimental General Relativity, Gravitation and Relativistic Field Theories (eds. R. H.Kleinert and R. T. Jantzen, World Scientific, 2008), 1236-1238; arXiv: 1006.5682.

[7] A. A. Chernitskii, On unification of gravitation and electromagnetism in the framework of a generalrelativistic approach, Grav. Cosmol. 15, 151-153 (2009); arXiv: 0907.2114.

[8] A. Eddington, The Mathematical Theory of Relativity (Cambridge, 1924).

[9] A. Einstein, Zur allgemeinen Relativitätstheorie, Sitzungsber. preuss. Akad. Wiss., phys.-math., 3238. Kl., 1923.

[10] M. Born and L. Infeld. Foundation of the new field theory, Proc. Roy. Soc. A 144, 425-451 (1934).

[11] A. A. Chernitskii, Light beams distortion in nonlinear electrodynamics, J. High Energy Phys. 11, Paper 15 (1998); hep-th/9809175.

[12] A. A. Chernitskii, Mass, spin, charge, and magnetic moment for electromagnetic particle. In: XI Advanced Research Workshop on High Energy Spin Physics (DUBNA-SPIN-05) Proceedings, (eds. A. V. Efremov and S. V. Goloskokov, JINR, Dubna, 2006), 234-239; hep-th/0603040.

[13] A. A. Chernitskii, Linear waves around static dyon solution of nonlinear (Born-Infeld) electrodynamics, Hadronic Journal 29, 497-528 (2006); hepth/0602079. 